\title{
Fractal and multifractal properties of a family of fractal networks
}

\author{
Bao-Gen $\mathrm{Li}^{1}, \mathrm{Zu}-\mathrm{Guo} \mathrm{Yu}^{1,2 *}$ and $\mathrm{Yu}^{\mathrm{Zhou}}{ }^{1}$ \\ ${ }^{1}$ Hunan Key Laboratory for Computation and Simulation in Science and Engineering and \\ Key Laboratory of Intelligent Computing and Information Processing of Ministry of Education, \\ Xiangtan University, Xiangtan, Hunan 411105, China. \\ ${ }^{2}$ School of Mathematical Sciences, Queensland University of Technology, \\ GPO Box 2434, Brisbane, Q4001, Australia.
}

\begin{abstract}
In this work, we study the fractal and multifractal properties of a family of fractal networks introduced by Gallos et al. (Proc. Natl. Acad. Sci. U.S.A., 2007, 104: 7746). In this fractal network model, there is a parameter $e$ which is between 0 and 1 , and allows for tuning the level of fractality in the network. Here we examine the multifractal behavior of these networks, dependence relationship of fractal dimension and the multifractal parameters on the parameter $e$. First, we find that the empirical fractal dimensions of these networks obtained by our program coincide with the theoretical formula given by Song et al. ( Nat. Phys, 2006, 2: 275). Then from the shape of the $\tau(q)$ and $D(q)$ curves, we find the existence of multifractality in these networks. Last, we find that there exists a linear relationship between the average information dimension $<D(1)>$ and the parameter $e$.
\end{abstract}

Key words: Complex network, scale-free, multifractality, box covering algorithm.

PACS: 89.75.Hc, 05.45.Df, 47.53.+n

\section{Introduction}

Complex networks have caused extensive attention due to their close connection with so many real-world systems, such as the world-wide web, the internet, energy landscapes, and biological and social systems [1]. The fractality and percolation transition [2], fractal transition [3] in complex networks, and properties of a scale-free Koch networks [4-6] have turned to be hot topics in recent years.

Fractal analysis (using the fractal dimension) is a useful method to describe global properties of complex fractal sets [7-9]. Song et al. $[1,10]$ proposed an algorithm to calculate the fractal dimension of complex networks which can unfold their self-similar property. They mentioned that the box counting fractal analysis is an effective tool for the further study of complex networks. But the fractal

${ }^{*}$ Corresponding author, email: yuzg1970@yahoo.com 
analysis is not enough when the object studied can not be described by a single fractal dimension. It has been found that the multifractal analysis (MFA) is a powerful tool in both the theory and practice to describe the spatial heterogeneity of fractal object systematically [11,12]. The MFA was originally raised to handle turbulence data, and now it has been successfully applied in many fields, such as financial modelling [13,14], biological systems [15-24] and geophysical systems [25-31]. Lee and Jung [32] found that MFA is the best tool to describe the probability distribution of the clustering coefficient of a complex network. Furuya and Yakubo [33] analytically and numerically demonstrated the possibility that the fractal property of a scale-free network cannot be characterized by a single fractal dimension when the network takes a multifractal structure. Almost at the same time, Wang et al. [34] proposed a modified fixed-size box-counting algorithm to study the multifractal property of complex networks.

In this paper, we study the fractal and multifractal properties of a family of complex networks introduced by Gallos et al. [35]. In order to imitate the fractal property of many scale-free networks found in nature, Song et al. [36] developed a network model to describe the fractality of networks. The main characteristic of this model is the introduction of a parameter $e$ which could be used to control the original hubs whether continue to form connections between the nodes in the process of the growth of complex networks. The authors [36] pointed out that the parameter $e$ can be regarded as a level of fractality of the network. The network corresponds to a pure fractal network which is a pure fractal set (defined by Mandelbrot [7]) when $e=0$, and a pure small world network when $e=1$ [2]. Later on, Gallos et al. [35] proposed a generalized version of this network model.

In Section 2, we introduce the generalized version of the network model in Ref. [35] and some of its topological properties. In Section 3, we examine the fractal dimension of these networks . A new fixed-size box-counting algorithm for MFA of networks modified from the one proposed in Ref. [34] is given in Section 4. The multifractal properties of the model networks and their results are also given in this Section. Some conclusions are presented in Section 5.

\section{Network model}

A graph (or network) is a collection of nodes which denote the elements of a system, and links or edges which identify the relations or interactions among these elements. In this section, the algorithm of the generalized version of the network model in Ref. [35] is presented. The network could be obtained by a method described as follows.

First we give a real number $0 \leq e \leq 1$, and two positive integers $m$ and $x(x \leq m)$. In the generation $n=0$, we start with only two nodes and one edge between them. In order to get the network of the generation $n+1$, every endpoint of each edge $L$ in the network of the generation $n$ is attached to $m$ new nodes. Then we generate a random number $p$ which obeys the uniform distribution between 0 and 1 . If $0 \leq p<e$, each edge $L$ of the generation $n$ is kept and $x-1$ new edges are appended to connect pairs of the new nodes attached to the endpoints of $L$; otherwise, for each edge $L$ of the generation $n$, we add $x$ new edges matching new nodes at the ends of $L$ and remove $L$ (see Fig. 1). As shown in Fig. 2, if we take $e=0, m=2, x=2$, we add $m=2$ new nodes 


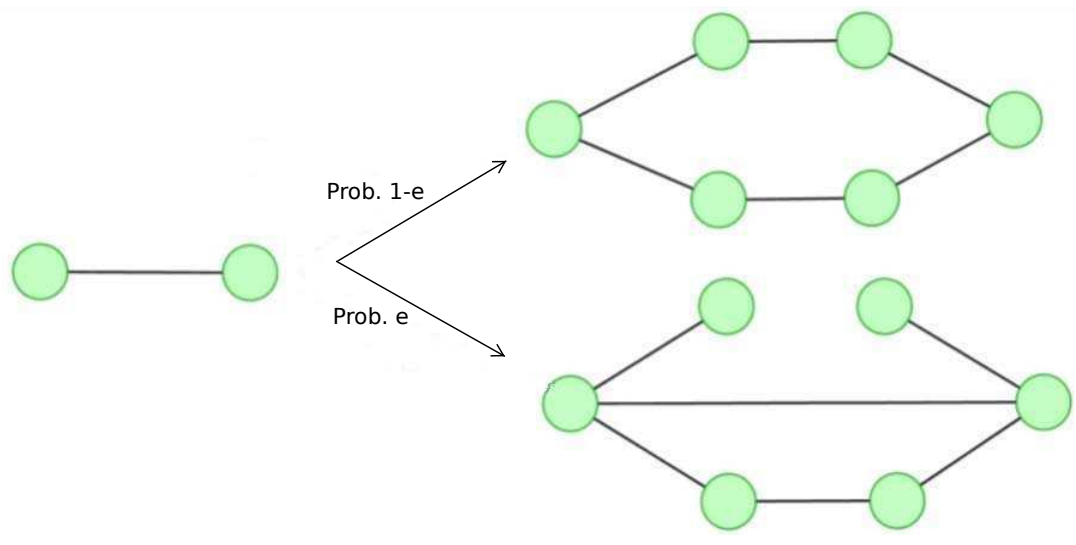

Figure 1: Construction of network. The link between hub remains with probability $e$, otherwise, it is replaced by another link between new nodes with probability $1-e$.

to the two endpoints of the sole edge in the generation $n=0$ to get the network of the generation $n=1$. Due to $e=0$, we take away the edge of $n=0$ and add $x=2$ edges between the new nodes. Notice that when $x=1$, we get tree structure without loop for any value of $e$.
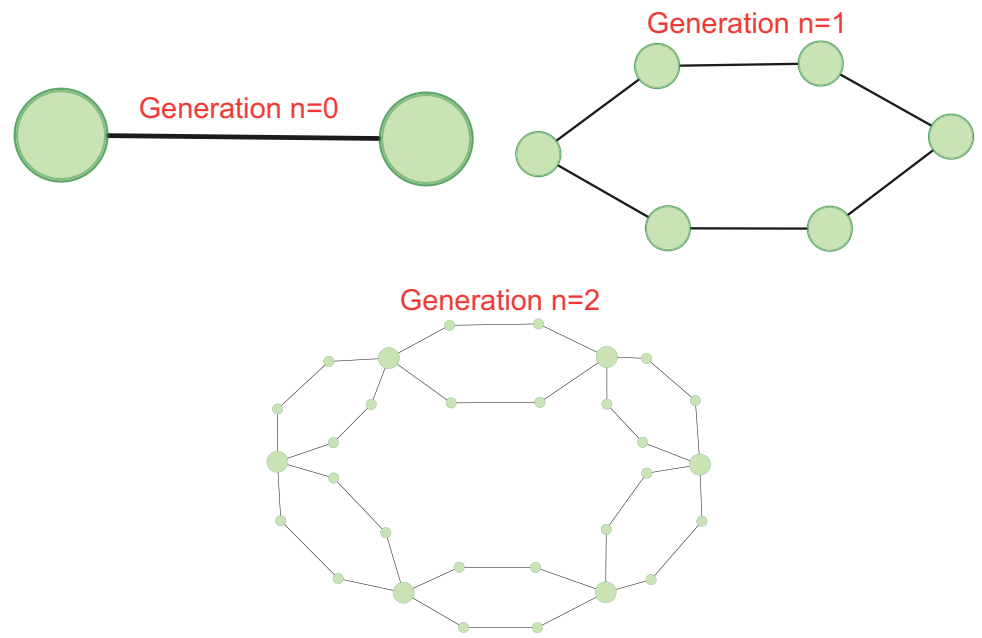

Figure 2: Construction of a pure fractal network. Example of the network modelof generations $n=0,1,2$ with parameters $m=2, x=2, e=0$.

According to above description, if we denote $M_{n}$ the number of edges in the network of the generation $n$, we can have $M_{n+1}=(2 m+x) * M_{n}$ in the generation $n+1$. Hence $M_{n}=(2 m+x)^{n}$. Meanwhile in the growth of the network from the generation $n-1$ to the generation $n$, each edge in the network of the generation $n-1$ produces $2 m$ new nodes. Hence we have $N_{n}=2 m M_{n-1}+N_{n-1}$, where $N_{n}$ is the number of nodes in the network of the generation $n$. Therefore,

$$
N_{n}=\frac{2 m}{2 m+x-1}(2 m+x)^{n}+2-\frac{2 m}{2 m+x-1}
$$

It was proved in Song et al. [36] that the degree distribution $P(k)$ of the network model satisfies a power law relationship $P(k) \approx k^{-\gamma}$ with $\gamma=1+\ln b / \ln s$, where $b$ is the scaling of the node number and $s$ is the scaling of the node degrees between two adjacent generations in the process of the 
growth of the network. From the algorithm described above, we know that if the degree of a node in the network of the generation $n$ is $K_{n}$, then it should be $m K_{n}+K_{n}$ with probability $e$, or $m K_{n}$ with probability $1-e$ in generation $n+1$, therefore $K_{n+1}=e\left(m K_{n}+K_{n}\right)+(1-e) m K_{n}=(m+e) K_{n}$. It is easy to know that $b=2 m+x$ from Eq. (1). So for the above model, we have

$$
\gamma=1+\frac{\ln (2 m+x)}{\ln (m+e)}
$$

In addition, a simple analysis shows that the clustering coefficient of the network model is 0 for any value of $e$.

\section{The fractal dimension}

We find that if the distance between two nodes of the generation $n$ is $L_{n}$, then in the network of the generation $n+1$, the distance $L_{n+1}$ would be $L_{n}$ with probability $e$, or $3 L_{n}$ with probability $1-e$. Hence $L_{n+1}=e L_{n}+3(1-e) L_{n}=(3-2 e) L_{n}$. From Ref. [36], we know that the theoretical fractal dimension of the model networks is $d_{f}^{T}=\ln b / \ln a$, where $a=L_{n+1} / L_{n}$. Therefore,

$$
d_{f}^{T}=\frac{\ln (2 m+x)}{\ln (3-2 e)}
$$

If $x=2, m=2$, we have $d_{f}^{T}=\ln (6) / \ln (3-2 e)$.

We can also numerically calculate the fractal dimension of the model networks using some algorithms (e.g. [10,37]). Here we adopt the random sequential box-counting algorithm proposed by Kim et al. [37] to estimate the fractal dimension of networks (two examples for estimating fractal dimension are shown in Fig. 3). We denote $d_{f}^{N}$ the fractal dimension of the network obtained numerically. First we want to check whether values of $d_{f}^{N}$ coincide with the theoretical values of fractal dimension $d_{f}^{T}$. If the numerical and theoretical fractal dimensions coincide with each other, we will have confidence on our process and program to estimate the multifractal curves $\tau(q)$ and $D(q)$ of these networks. Due to the limit of computational capacity of our computers, we only generate the networks up to the generation $n=5$.

For each value of $e$, we generate 100 networks (100 realizations) and calculate the average value of $d_{f}^{N}$ over the 100 realizations. The $e$ vs $\left\langle d_{f}^{N}>\right.$ plot is presented in Fig. 4. From Fig. 4, we can see that the numerical $\left\langle d_{f}^{N}>\right.$ coincides with the theoretical $<d_{f}^{T}>$ perfectly.

\section{Multifractal analysis}

In this section, we first introduce a new algorithm for MFA of networks modified from the one proposed in Ref. [34] and then apply it to the model networks presented in Section 2.

Two networks which have the same fractal dimension may look completely different. In addition, when the networks have rich scale and self-similar structures, they exhibit different dimensions in different scales. MFA is a powerful method to study the networks with such characteristics.

At present, the fixed-size box-counting algorithm is the most common algorithm for MFA [12]. For a given probability measure $0 \leq \mu \leq 1$ with support set $E$ in metric space, we consider the 


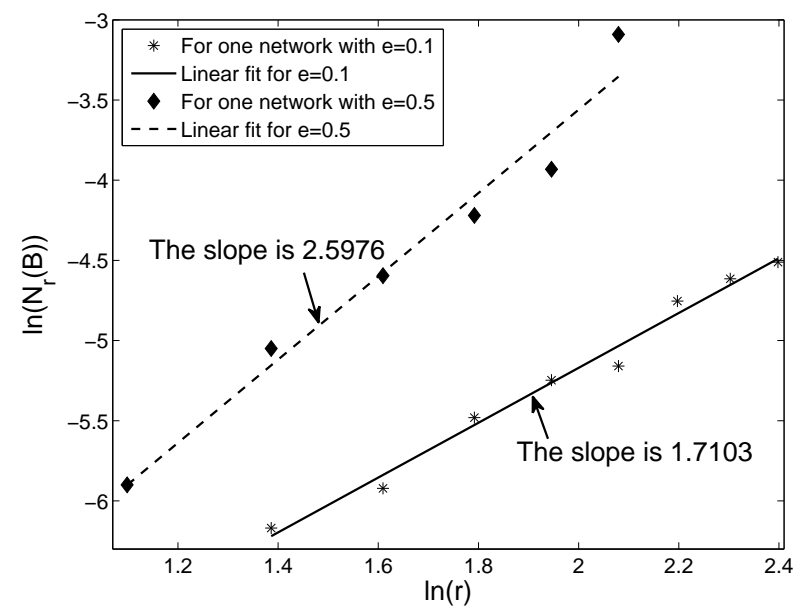

Figure 3: Two examples to estimate the fractal dimension of networks for $e=0.1$ and 0.5 , here parameters $n=5, m=2, x=2$. We can see the estimated fractal dimension is very close to the theoretical result 2.5850 (for $e=0.5$ ) and 1.7402 (for $e=0.1$ ) respectively.

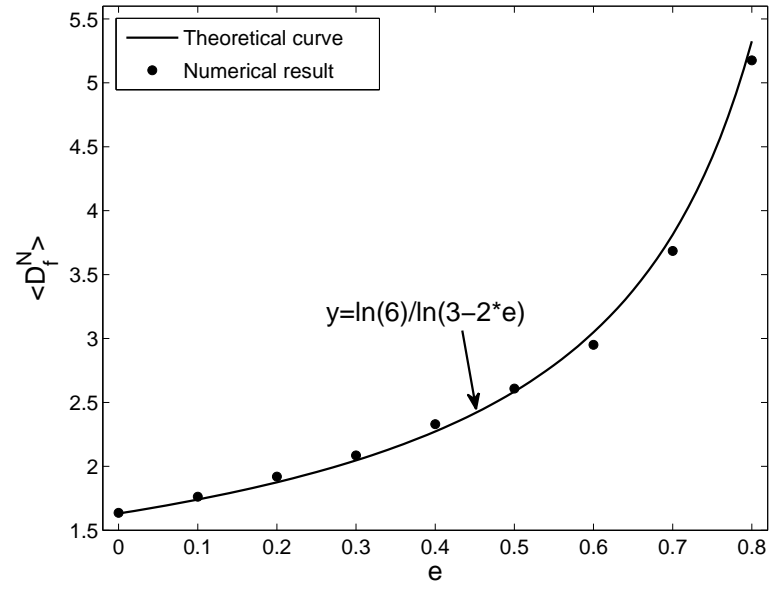

Figure 4: Numerical result of the relationship between the fractal dimension $\left\langle d_{f}^{N}\right\rangle$ of the networks and $e$ with parameters $n=5, m=2, x=2$. Here $<\cdot>$ means the average over 100 realizations. 
partition function

$$
Z_{r}(q)=\sum_{\mu(B) \neq 0}[\mu(B)]^{q}
$$

where $q \in R$, the result is the sum of all the different non-overlapping boxes $B$ with a given size $r$ in the covering of the support set $E$. It is easy to know that $Z_{r}(q) \geq 0$ and $Z_{r}(0)=1$. We define the mass exponent function $\tau(q)$ of the measure $\mu$ as

$$
\tau(q)=\lim _{r \rightarrow 0} \frac{\ln Z_{r}(q)}{\ln r}
$$

Then we get the generalized fractal dimensions of the measure $\mu$ by

$$
D(q)=\frac{\tau(q)}{q-1}, q \neq 1
$$

and

$$
D(1)=\lim _{r \rightarrow 0} \frac{Z_{(1, r)}}{\ln r}, q=1
$$

where $Z_{(1, r)}=\sum_{\mu(B) \neq 0} \mu(B) \ln \mu(B)$.

In practice, the generalized fractal dimensions are usually obtained by linear regression. Specifically, $D(0)$ is the fractal dimension of the support set of the measure $\mu, D(1)$ and $D(2)$ are called the information dimension and the correlation dimension respectively.

For a network, the measure $\mu$ of each box can be defined as the ratio of the number of nodes covered by the box to the total number of nodes in the network [34,37]. We need to complete the following two steps before we proceed MFA.

i) Map a network to an adjacent matrix $A_{N \times N}$, where $N$ is the total number of nodes in the network. It is easy to know that $A_{N \times N}$ is a symmetric matrix where the elements $a_{i j}=1$ when there is an edge between the nodes $i$ and $j$, otherwise $a_{i j}=0$. Here, the edge from node $i$ to node $i$ is not considered, so $a_{i i}=0$.

ii) Use $A_{N \times N}$ to calculate the shortest distance between any two nodes in the network and store them into another matrix $B_{N \times N}$. Here, in our study, we use Dijkstras algorithm of MatLab toolbox to calculate the shortest distance between two nodes in the network.

After finishing the two steps presented above, we can use matrix $B_{N \times N}$ as the input of MFA of the network model described in Section 2 based on a new algorithm for MFA of networks modified from the one proposed in Ref. [34] as follows.

(I) Ensure that all nodes in the network are not covered, and no node has been selected as the center of a box.

(II) According to the size of our networks $N=6222$ (with the parameters $n=5 ; m=2, x=2$ ), we set $t=1,2, \ldots, T$. Here we take $T=1000$, then we rearrange the nodes number into $T=1000$ different random orders. That is to ensure that the nodes of a network are randomly chosen as center nodes.

(III) Set the radius $r$ of boxes which will be used to cover the nodes in the range $[1, d]$, where $d$ is the diameter of the network (i.e. the longest distance between nodes in the network). 
(IV) Treat the nodes of the $t$ th kind of random orders that we have got in (II) as the center of a box successively, then search all the other nodes. If a node has a distance to the center node within $r$ and has not been covered yet, then cover it.

(V) If no more new nodes can be covered by this box, then we abandon this box.

(VI) Repeat (IV) - (VI) until all the nodes are covered by the corresponding boxes. We denote the number of boxes in this box covering as $N(t, r)$.

(VII) Repeat steps (III) and (VI) for all the random orders to find a box covering with minimal number of boxes $N(t, r)$.

(VIII) For each nonempty box $B$ in the first box covering with minimal number of boxes, we define its measure as $\mu(B)=N_{B} / 6222$, where $N_{B}$ is the number of nodes covered by the box $B$. For each $r$, we calculate the partition sum $Z_{r}(q)=\sum_{\mu(B) \neq 0}[\mu(B)]^{q}$.

(IX) For different $r$, we repeat (III)-(VIII). Then we use $Z_{r}(q)$ for linear regression.

Remark 1: In the algorithm of MFA of networks proposed in Ref. [34], we use $\bar{Z}_{r}(q)$ (the average of $Z_{r}(q)$ for all $T=1000$ different random orders of the nodes) for linear regression to get $\tau(q)$ (hence $D(q)$ ). But when $q=0, D(0)$ got in this way is not the box-counting dimension of the network because there requires minimum number of boxes which cover the fractal set (network here) [9]. Here we modify to use $Z_{r}(q)$ of a covering with minimum number of boxes for linear regression to get $\tau(q)$ (hence $D(q)$ ). So when $q=0, D(0)$ is exact the box-count dimension of the network. It is a more reasonable extension from the traditional MFA.

In order to get the range $r \in\left[r_{\min ,} r_{\max }\right]$ in which the networks obey the power law and then to get the mass exponents $\tau(q)$ and the generalized fractal dimensions $D_{q}$, linear regression is an important step. In our calculation, we run the linear regression of $\ln Z_{r}(q)$ against $\ln r$ to get $\tau(q)$, and then get $D(q)$ through formula $D(q)=\tau(q) /(q-1)$ for $q \neq 1$ and $D(1)$ through the linear regression of $Z_{(1, r)}=\sum_{\mu(B) \neq 0} \mu(B) \ln \mu(B)$ against $\ln r$ for $q=1$.

By applying the new fixed-size box-counting algorithm described above on the model networks, we get the following results:

First, for each value of $e$ (here we take $e=0.1,0.2, \ldots, 0.8$ ), we generate 100 networks (we take 100 realizations because the MFA for networks is very time consuming when the network is large), and calculate the $\tau(q)$ and $D(q)$ curves for each network using the new fixed-size box-counting algorithm. Then we take average for these $\tau(q)$ and $D(q)$ curves over the 100 realizations. The shape of the $\langle\tau(q)\rangle$ curves shown in Fig. 5 and the $\left\langle D_{q}\right\rangle$ curves shown in Fig. 6 are all nonlinear, which indicate that all the networks we studied have multifractal property. We also find that the value of $\Delta(<D(q)>)$ defined by $\max (<D(q)>)-\min (<D(q)>)$ increases with the increase of the parameter $e$, which indicates that the multifractal property of the model networks becomes more obvious when the value of the parameter $e$ becomes larger.

The multifractal property of the model networks revealed by our work indicates that the model networks are very complicated which cannot be characterized by a single fractal dimension. The MFA algorithm proposed here can be used to provide a more accurate characterization for the model networks, even for some other complicated networks. 


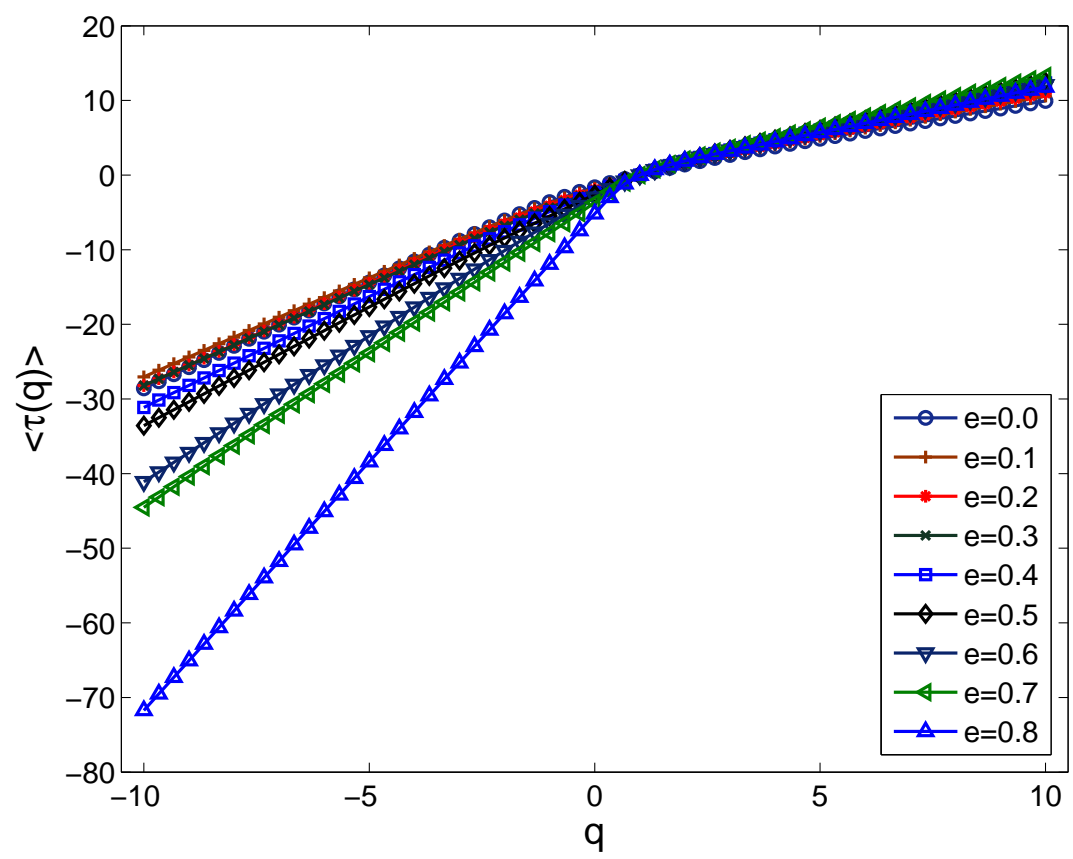

Figure 5: The $\tau(q)$ curves of the network model, here $<\cdot>$ means the average over 100 realizations.

Second, we find that the average information dimension $\langle D(1)>$ has a linear relation with the parameter $e$, i.e. $\langle D(1)\rangle=1.5053 * e+1.4735$ as shown in Fig. 7 , which is different from that of $D(0)$ shown in Eq. (3).

Remark 2: Furuya and Yakubo [33] also proposed an algorithm for MFA of complex networks. The difference between the algorithm in Ref. [33] and our algorithm is the definition of the measure $\mu$. In the algorithm in Ref. [33], it allows that any two boxes in the box covering have overlap and defines the measure $\mu_{i}$ by counting the times of overlaps of each node, hence it is not a natural extension of the traditional MFA (see Eq. (4)). In our algorithm, overlap of any two boxes in the box covering is not allowed, so it is a natural extension of the traditional MFA. Our network model with $e>0$ is different from the $(u, v)$-flower network model. Only the network model with $e=0$ corresponds to the deterministic $(u, v)$-flower network model with $u=v=3$. Furuya and Yakubo [33] also gave a theoretical formula for the $\tau(q)$ function of $(u, v)$-flower network model (Eq. (11) of Ref. [33]). When $u=v=3, \tau(q)$ has the formula [33]: $\tau(q)=q$ if $q \geq \ln (6) / \ln (2)=2.5850$, and $\tau(q)=(q-1) \frac{\ln (6)}{\ln (3)}$ if $q<\ln (6) / \ln (2)=2.5850$. We compared this formula with our numerical result for $e=0$ in Fig.5, and found that we also have $\tau(q) \simeq q$ if $q \geq 2.5850$, but the $\tau(q)$ values are different from $(q-1) \frac{\ln (6)}{\ln (3)}$ if $q<2.5850$.

\section{Conclusion}

We have studied the fractal and multifractal properties of a family of model networks that were originally proposed to explain the origin of fractality in complex networks. This model introduces a parameter $e$, which can be used to tune the fractality level of the network. One can get a pure fractal network when $e=0$ and obtain a small-world network when $e=1$. We investigated the 


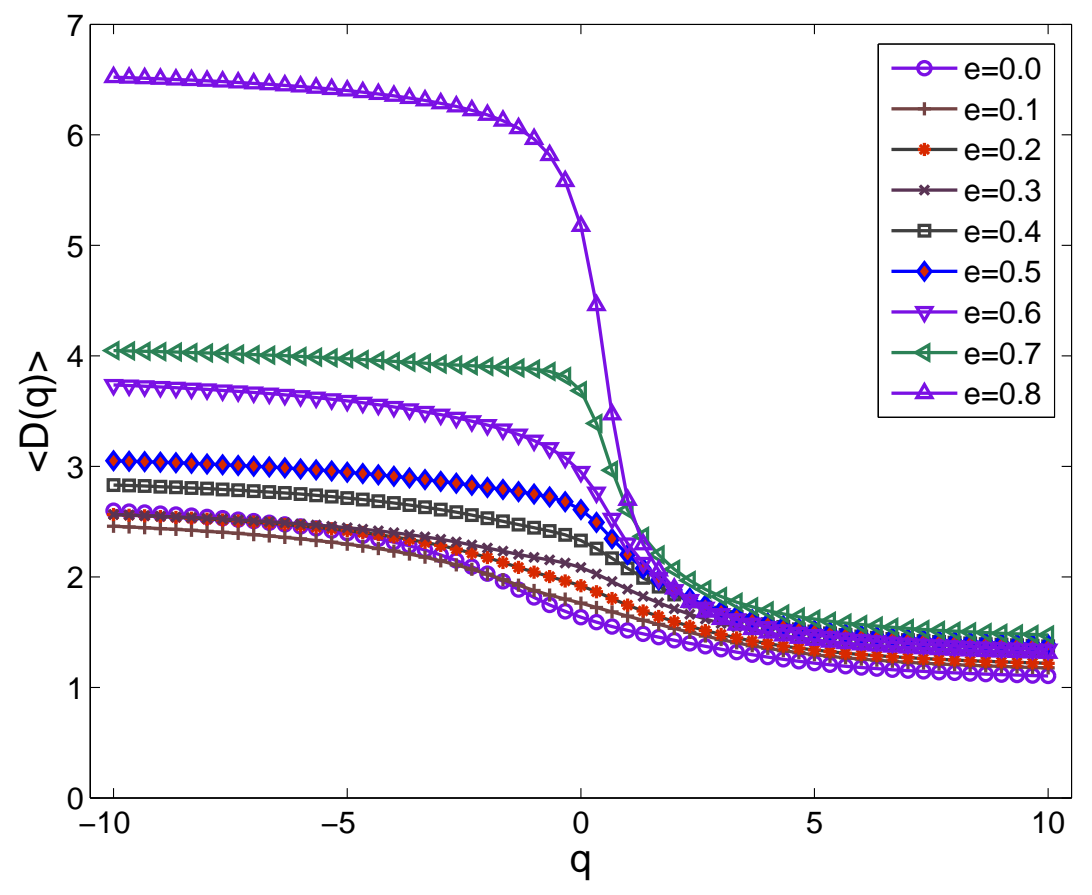

Figure 6: The $D(q)$ curves of the network model, here $<\cdot>$ means the average over 100 realizations.

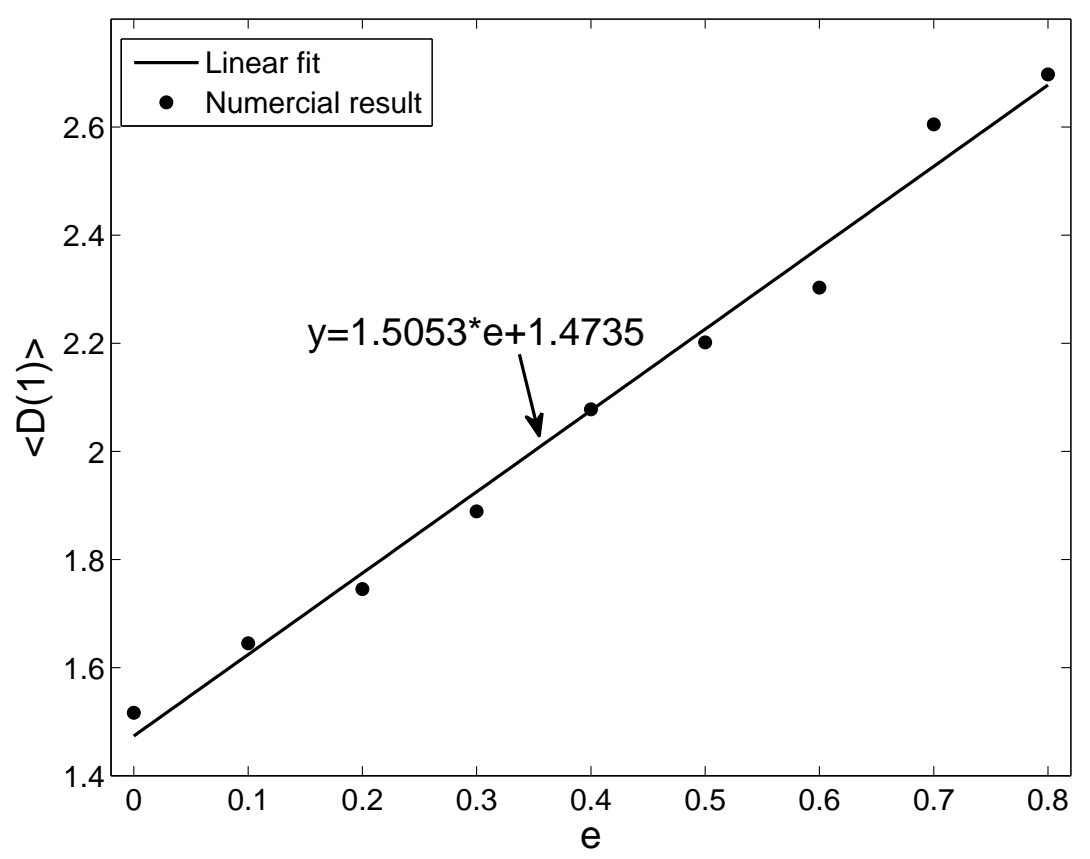

Figure 7: The relationship of $\langle D(1)>$ against parameter $e$ respectively, here $\langle\cdot\rangle$ means the average over 100 realizations. 
fractal and multifractal properties through numerical calculation. To make the calculation feasible and accurate, we calculated the model with parameters $n=5 ; m=2, x=2 ; e=0,0.1,0.2, \ldots, 0.8$; $q=-10, \ldots, 10$. The result of the $\tau(q), D(q)$ (including $D(0)$ and $D(1)$ ) are averaged over 100 realizations (networks). The shape of $\tau(q)$ and $D(q)$ curves are all nonlinear, which indicates that all the networks we studied have multifractal property. We also found that the value of $\Delta(<D(q)>)=\max (<D(q)>)-\min (<D(q)>)$ increases with the increase of the parameter $e$, which indicates that the multifractal property of the model becomes more obvious when the value of the parameter $e$ becomes larger.

We also found that the average information dimension $\langle D(1)>$ has a linear relation with the parameter $e$, i.e. $\langle D(1)\rangle=1.5053 * e+1.4735$.

The MFA algorithm proposed here can be used to provide a more accurate characterization for the model networks, even for some other complicated networks.

\section{Acknowledgments}

This project was supported by the Natural Science Foundation of China (Grant Nos. 11071282 and 11371016), the Chinese Program for Changjiang Scholars and Innovative Research Team in University (PCSIRT) (Grant No. IRT1179); the Research Foundation of Education Commission of Hunan Province of China (Grant No. 11A122); the Lotus Scholars Program of Hunan province of China. The authors would like to thank the editor and the reviewers for their insights, comments and suggestions to improve this paper.

\section{References}

[1] Song C, Havlin S and Makse H A, Self-similarity of complex networks, Nature 433 (2005) 392-395.

[2] Rozenfeld H D and Makse H A, Fractality and the percolation transition in complex networks, Chem. Eng. Sci. 64 (2009) 4572-4575.

[3] Rozenfeld H D, Song C and Makse H A, Small-World to Fractal Transition in Complex Networks: A Renormalization Group Approach, Phys. Rev. Lett. 104 (2010) 025701.

[4] Zhang Z., Zhou S., Zou T. and Chen G.,Fractal scale-free networks resistant to disease spread, J. Stat. Mech.: Theor. Exp. 9 (2008) P09008.

[5] Zhang Z, Zhou S, Xie W, Chen L, Lin Y, and Guan J, Standard random walks and trapping on the Koch network with scale-free behavior and small-world effect, Phys. Rev. E 79 (2009) 061113.

[6] Liu J X and Kong X M, Establishment and structure properties of the scale-free Koch network (in Chinese), Acta Phy. Sin. 59 (2010) 2244-2249.

[7] Mandelbrot B B, 1983 The Fractal Geometry of Nature (New York: Academic Press).

[8] Feder J, 1988 Fractals (New York: Plenum).

[9] Falconer K, 1997 Techniques in Fractal Geometry (New York: Wiley). 
[10] Song C, Gallos L K, Havlin S and Makse H A, How to calculate the fractal dimension of a complex network: the box covering algorithm, J. Stat. Mech.: Theor. Exp. 3 (2007) P03006.

[11] Grassberger P and Procaccia I, Characterization of Strange Attractors, Phys. Rev. Lett 50 (1983) 346-349.

[12] Halsey T C, Jensen M H, Kadanoff L P, Procaccia I and Shraiman B I, Fractal measures and their singularities: The characterization of strange sets, Phys. Rev. A 33 (1986) 1141-1151.

[13] Canessa E, Multifractality in time series, J. Phys. A 33 (2000) 3637-3651.

[14] Anh V V, Tieng Q M and Tse Y K, Cointegration of stochastic multifractals with application to foreign exchange rates, Int. Trans. Oper. Res. 7 (2000) 349-363.

[15] Yu Z G, Anh V and Lau K S, Multifractal characterisation of length sequences of coding and noncoding segments in a complete genome, Physica A 31 (2001) 351-361.

[16] Yu Z G, Anh V and Lau K S, Measure representation and multifractal analysis of complete genomes, Phys. Rev. E 64 (2001) 031903.

[17] Yu Z G, Anh V and Lau K S, Multifractal and correlation analyses of protein sequences from complete genomes, Phys. Rev. E 68 (2003) 021913.

[18] Yu Z G, Anh V and Lau K S, Chaos game representation of protein sequences based on the detailed HP model and their multifractal and correlation analyses, J. Theor. Biol. 226 (2004) 341-348.

[19] Yu Z G, Anh V V, Lau K S and Zhou L Q, Clustering of protein structures using hydrophobic free energy and solvent accessibility of proteins, Phys. Rev. E 73 (2006) 031920.

[20] Yu Z G, Xiao Q J, Shi L, Yu J W, and Anh V, Chaos game representation of functional protein sequences, and simulation and multifractal analysis of induced measures, Chin. Phys. B 19 (2010) 068701.

[21] Anh V V, Lau K S and Yu Z G, Recognition of an organism from fragments of its complete genome, Phys. Rev. E 66 (2002) 031910.

[22] Zhou L Q,Yu Z G, Deng J Q, Anh V and Long S C, A fractal method to distinguish coding and non-coding sequences in a complete genome based on a number sequence representation, J. Theor. Biol. 232 (2005) 559-567.

[23] Han J J and Fu W J, Wavelet-based multifractal analysis of DNA sequences by using chaos-game representation, Chin. Phys. B 19 (2010) 010205.

[24] Zhu S M, Yu Z G, Ahn V, Protein structural classification and family identification by multifractal analysis and wavelet spectrum, Chin. Phys. B 20 (2011) 010505.

[25] Kantelhardt J W, Koscielny-Bunde E, Rybski D, Braun P, Bunde A and Havlin S, Long-term persistence and multifractality of precipitation and river runoff records, J. Geophys. Res. 111 (2006) D01106.

[26] Veneziano D, Langousis A and Furcolo P, Multifractality and rainfall extremes: A review, Water Resour. Res. 42 (2006) W06D15.

[27] Venugopal V, Roux S G, Foufoula-Georgiou E and Arneodo A, Revisiting multifractality of high-resolution temporal rainfall using a wavelet-based formalism, Water Resour. Res. 42 (2006) W06D14.

[28] Yu Z G, Anh V V, Wanliss J A, and Watson S M, Chaos game representation of the Dst index and prediction of geomagnetic storm events, Chaos, Solitons and Fractals 31 (2007) 736-746.

[29] Yu Z G, Anh V and Eastes R, 2009 J. Geophys. Res. 114 A05214 
[30] Yu Z G, Anh V, Wang Y, Mao D and Wanliss J, Multifractal analysis of geomagnetic storm and solar flare indices and their class dependence, J. Geophys. Res. 115 (2010) A10219

[31] Zang B J and Shang P J, Multifractal analysis of the Yellow River flow, Chin. Phys. 16 (2007) 565-569.

[32] Lee C Y and Jung S H, Statistical self-similar properties of complex networks, Phys. Rev. E 73 (2006) 066102.

[33] Furuya S and Yakubo K, Multifractality of complex networks, Phys. Rev. E 84 (2011) 036118

[34] Wang D L, Yu Z G and Anh V, Multifractal analysis of complex networks, Chin. Phys. B 21 (2012) 080504.

[35] Gallos L K, Song C, Havlin S and Makse H A, Proc. Natl. Acad. Sci. U.S.A. 104 (2007) 7746-7751.

[36] Song C, Havlin S and Makse H A, Origins of fractality in the growth of complex networks, Nat. Phys. 2 (2006) $275-281$.

[37] Kim J S, Goh K I, Salvi G, Oh E, Kahng B and Kim D, Fractality in complex networks: Critical and supercritical skeletons, Phys. Rev. E 75 (2007) 016110. 